\title{
HI IMAGING OF RADIO ACTIVE ELLIPTICALS
}

\author{
J.H. van Gorkom \\ Princeton University Observatory \\ Peyton Hall \\ Princeton, NJ 08544 \\ USA
}

ABSTRACT. Statistics on HI absorption in radio galaxies suggest that accretion of gas into the center might be a general phenomenon. Images of the HI emission from these galaxies show that the gas must have an external origin. The rotation curves derived from the $\mathrm{HI}$ data are flat.

High resolution observations of $\mathrm{HI}$ absorption against the unresolved radio cores exist for 4 radio galaxies. All show absorption at velocities redshifted with respect to the systemic velocities, by 100 to $400 \mathrm{~km} / \mathrm{s}$. Three show absorption at the systemic velocity as well, none shows blueshifted absorption. Examples are shown in Fig. 1 for NGC 1052 (van Gorkom et al, 1986) and NGC 5363 (van Gorkom and Laing, in prep.). The high velocity gas is only seen in absorption against the cores, not against extended structures, thus it must be close to the center. Either the gas is falling in or it is on non-circular orbits, if that is true no preference should be found for redshifted gas in a larger sample.

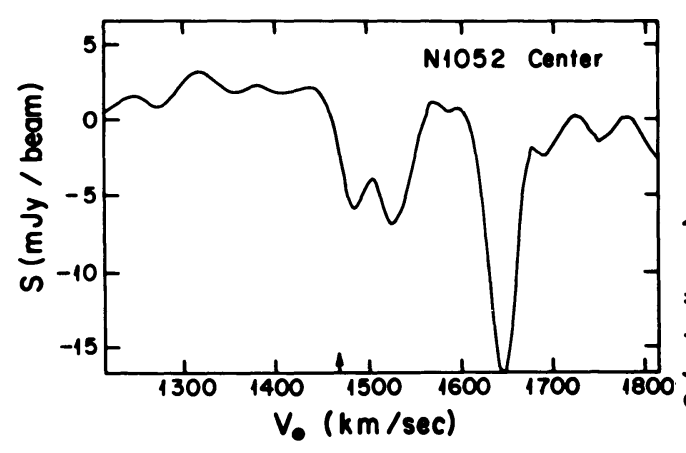

Figure 1. Absorption profiles for NGC 5363 and NGC 1052. The arrow indicates the systemic velocity of the galaxies. Note the absorption at high velocities.

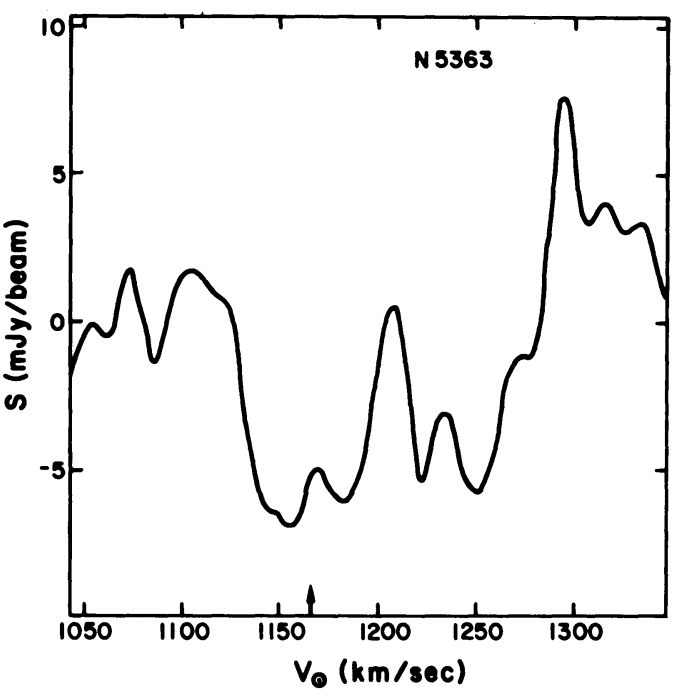

421

T. de Zeeuw (ed.), Structure and Dynamics of Elliptical Galaxies, 421-422.

(C) 1987 by the IAU. 
An overlay of the total HI emission on an optical photograph is shown in Fig. 2 for NGC 1052 and 5128 (van Gorkom et.al., 1986, 1987). The galaxies are very similar: the $\mathrm{HI}$ has settled into a regularly rotating disk, but the rotation axes of gas and stars are almost perpendicular and the specific angular momentum of the gas is much larger than of the stars. The gas in the outer parts is not on stable orbits as can be inferred from the direction of rotation of gas and stars and the orientation of the warps. In the inner parts gas on non-circular orbits is seen, in NGC 1052 it is a regular flow pattern, showing up in emission and absorption, while in NGC 5128 it looks like individual clouds falling in. The gas to dust ratios are close to galactic for both galaxies.

From the above we conclude that the gas must have an external origin, as opposed to stellar mass loss. Most likely it comes from another galaxy, since the gas to dust ratios are normal.

The rotation curves (Fig. 3) stay flat out to the last measured point, giving an M/L of 15 at $16 \mathrm{kpc}$ and of 5 at $4 \mathrm{kpc}$ for NGC 1052 and 5128 respectively. A comparison with the mass of NGC 5128 as derived from X-ray observations leads to a discrepancy of a factor 4 (Knapp, 1987).

\section{REFERENCES}

Knapp, G.R., 1987. This volume, p. 145.

van Gorkom, Knapp, Raimond, Faber, Gallagher, 1986. A. J., 91, 791 van Gorkom, van der Hulst, Haschick, Tubbs, 1987. in preparation

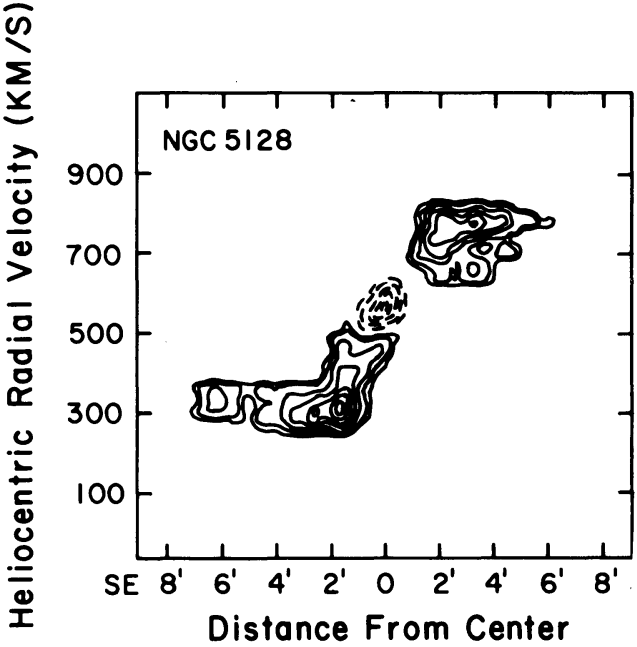

Figure 2. The total HI emission of NGC 5128 at top, NGC 1052 at right. Figure 3. An HI rotation curve not corrected for inclination (above).

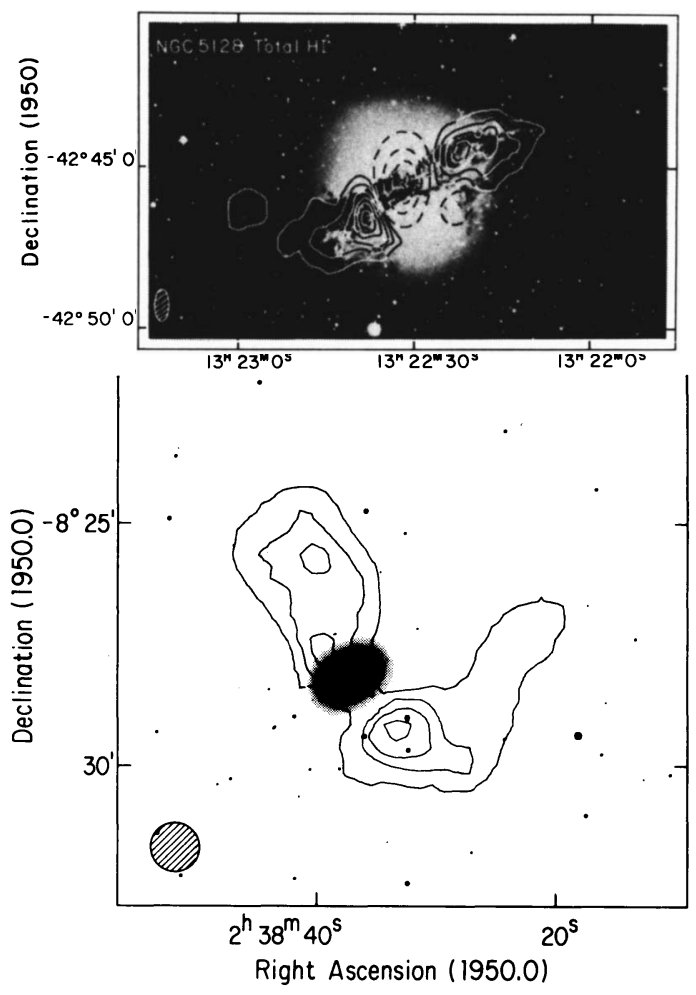

